\title{
One Dimensional Random Motion on Segment with Reflecting Edges and Dependent Increments
}

\author{
Gurami Tsitsiashvili \\ IAM FEB RAS, FEFU, Vladivostok, Russia \\ Email: guram@iam.dvo.ru
}

How to cite this paper: Tsitsiashvili, G. (2018) One Dimensional Random Motion on Segment with Reflecting Edges and Dependent Increments. Journal of Applied Mathematics and Physics, 6, 488-497. https://doi.org/10.4236/jamp.2018.63045

Received: February 1, 2018

Accepted: March 12, 2018

Published: March 15, 2018

Copyright $\odot 2018$ by author and Scientific Research Publishing Inc. This work is licensed under the Creative Commons Attribution International License (CC BY 4.0).

http://creativecommons.org/licenses/by/4.0/

\section{(c) (i) Open Access}

\begin{abstract}
In previous papers, the author considered the model of anomalous diffusion, defined by stable random process on an interval with reflecting edges. Estimates of the rate convergence of this process distribution to a uniform distribution are constructed. However, recent physical studies require consideration of models of diffusion, defined not only by stable random process with independent increments but multivariate fractional Brownian motion with dependent increments. This task requires the development of special mathematical techniques evaluation of the rate of convergence of the distribution of multivariate Brownian motion in a segment with reflecting boundaries to the limit. In the present work, this technology is developed and a power estimate of the rate of convergence to the limiting uniform distribution is built.
\end{abstract}

\section{Keywords}

Fractional Brownian Motion, Rate of Convergence, Anomalous Diffusion, Segment with Reflecting Edges

\section{Introduction}

In recent years, fractional Brownian motion has experienced significant growth in the applied problems of physics [1] [2] [3] [4] in connection with the necessity of modelling chaotic behaviour of the diffusing impurity in a variety of environments and alloys. Therefore there is a need to analyse the speed of mixing of impurities (convergence to the uniform distribution) in areas with reflecting boundaries, which cannot be obtained by the method of Fourier series.

Algorithm of constructing of such estimates was described in [5] for one-dimensional case and was based on the method of analysis of anomalous 
diffusion [6], simulating stable random process with independent increments. It is based on reflection formula for the density of the anomalous diffusion process. However, in recent years, there have been a lot of physical researches, in which models of fractional Brownian motions are used.

Therefore, in the present work the algorithm of the corresponding estimates for the fractional Brownian motion on interval with reflecting edges is constructed. This algorithm is based on a calculation of a derivative of series which is describing density of fractional Brownian motion distribution [7].

\section{Preliminaries}

Let $y(t), t \geq 0$ is a random process with a fixed initial value $y(0)=0$. Consider random process $Y(t)$ comparable to $y(t)$ but reflected at the ends of the segment $[0,1]$ in the following way.

\section{Construction of random process reflected on interval $[0,1]$}

The one-dimensional process $y=y(t), t \geq 0$ is mapped to the reflected (from the boundaries of the segment [0,1] random process $Y(t)=g(s(y(t)))$, where the functions $s: E^{1} \rightarrow[0,2), g:[0,2) \rightarrow[0,1]$ are defined by the equalities $s(u)=(u) / \bmod 2, g(u)=u, 0 \leq u \leq 1, \quad g(u)=2-u, 1<u<2$, [5]. Here $u / \bmod A=A\{u / A\}, A>0 ;\{z\}$-the fractional part of a real number $z$.

\section{Reflection formula for random process with symmetric density}

Let $f_{t}=f_{t}(u)$ is a distribution density of a random variable (r.v.) $Y(t)$. Then by the formula $Y(t)=g(s(y(t)))$ we have:

$$
f_{t}(u)=\sum_{k=-\infty}^{\infty} p_{t}(u-2 k)+\sum_{k=-\infty}^{\infty} p_{t}(2-(1+u)-2 k), u \in[0,1],
$$

$f_{t}(u)=0, u \notin[0,1]$. If for each $t>0$ the density $p_{t}(u)$ is symmetric in $u: p_{t}(u)=p_{t}(-u)$, then for $u \in[0,1]$

$$
f_{t}(u)=\sum_{k=-\infty}^{\infty} p_{t}(u-2 k)+\sum_{k=-\infty}^{\infty} p_{t}(-u-(2 k+1))=\sum_{k=-\infty}^{\infty} p_{t}(u-k),
$$

$f_{t}(u)=0, u \notin[0,1]$.

It is interesting to note that the formula (1), giving the distribution of the reflected diffusion process, is very similar by its structure to the formula obtained by reflection ([8], Chapter III, $\S 13$, paragraphs 5,6$)$ and gives the solution of the wave equation for a finite string with fixed ends.

\section{Reflection formula for random process with periodic initial conditions}

Define $f_{t}(u-a)$ the density of distribution of random process $y(t)+a$ reflected from the ends of the segment $[0,1], 0 \leq a \leq 1$. Let $S$ is a random variable, uniformly distributed on the set $\{0,1 / n, \cdots,(n-1) / n\}$, and random variable $S$ and random process $y(t)$ are independent. We introduce the function $F_{t}(u)=f_{t}(u-S), 0 \leq u \leq 1$, then

$$
\begin{aligned}
F_{t}(u) & =\frac{1}{n} \sum_{s=0}^{n-1} f_{t}(u-s / n)=\frac{1}{n} \sum_{s=0}^{n-1} \sum_{k=-\infty}^{\infty} p_{t}(u-k-s / n) \\
& =\frac{1}{n} \sum_{k=-\infty}^{\infty} p_{t}(u-k / n)
\end{aligned}
$$


Because of Formula (2) the function $F_{t}(u)$ possesses following properties:

$$
\begin{gathered}
F_{t}(u)=F_{t}(u+1 / n), 0 \leq u \leq 1-1 / n, \\
F_{t}(u / n)=\frac{1}{n} \sum_{k=-\infty}^{\infty} p_{t}((u-k) / n), 0 \leq u \leq 1 .
\end{gathered}
$$

The first equality in (3) means that the function $F_{t}(u)$ consists of $n$ periods of length $1 / n$ on the interval $[0,1]$. The second equality in (3) means that on the interval $[0,1 / n]$ the function $F_{t}(u)$ characterizes the distribution density of a random process $n y(t)$ reflected from the ends of the segment $[0,1 / n]$.

In turn, the function $F_{t}(u-1 / 2 n)$ characterizes the distribution density of a random process $y(t)+\hat{s}+1 / 2 n$ with an initial condition $\hat{s}+1 / 2 n$, which has a uniform distribution on the set of points $1 / 2 n, 3 / 2 n, \cdots,(2 n-1) / 2 n$ and is independent with random process $y(t)$.

Self-similar stochastic processes with reflection and periodic initial conditions

Let the random process $y(t)$ is self-similar of order $a$ [9], i.e. for every $t \geq 0$ the random variables $y\left(t / r^{-1 / a}\right), r y(t)$ coincides in distribution:

$$
y\left(t / r^{-1 / a}\right) \stackrel{d}{=} r y(t)
$$

In terms of the density distribution this relation looks like

$$
p_{t}(u r)=p_{t / r^{-1 / a}}(u) \text {. }
$$

We now turn to the calculation of the function $F_{t}(u / n)$ assuming that selfsimilar random process $y(t)$ has a symmetric density $p_{t}(u)$ :

$$
\begin{aligned}
F_{t}(u / n) & =\sum_{k=-\infty}^{\infty} \frac{1}{n} p_{t}((u-k) / n) \\
& =\sum_{k=-\infty}^{\infty} p_{t / n^{1 / a}}(u-k)=f_{t / n^{1 / a}}(u), 0 \leq u \leq 1 .
\end{aligned}
$$

Hence in particular it follows the equality

$$
F_{t}(u / n-1 / 2 n)=f_{t / n^{-1 / a}}(u-1 / 2), 0 \leq u \leq 1
$$

Then from Formulas (3), (5) we get the equality

$$
F_{t}(u-1 / 2 n)=f_{t n^{1 / a}}(u-1 / 2) .
$$

\section{Multidimensional random process with independent components}

For simplicity of notation all future constructions without loss of generality, we spend for the flat case $m=2$. Consider a two-dimensional random process with independent components of $\vec{y}(t)=\left(y_{1}(t) y_{2}(t)\right)$, having symmetric and self-similar density distribution of order $a$. Construct a process $\vec{Y}(t)$ with reflections from the boundaries of the square $[0,1]^{2}$ using the obvious equalities:

$$
\vec{Y}(t)=\left(Y_{1}(t), Y_{2}(t)\right), Y_{1}(t)=g\left(s\left(y_{1}(t)\right)\right), Y_{2}(t)=g\left(s\left(y_{2}(t)\right)\right) .
$$

In this case equalities $p_{t}\left(u_{1}, u_{2}\right)=p_{t}\left(u_{1}\right) p_{t}\left(u_{2}\right)$ are true and so 


$$
\begin{aligned}
f_{t}\left(u_{1}, u_{2}\right)= & \sum_{k_{1}, k_{2}=-\infty}^{\infty}\left[\left(p_{t}\left(u_{1}-2 k_{1}, u_{2}-2 k_{2}\right)+p_{t}\left(2-\left(1+u_{1}\right)-2 k_{1}, u_{2}-2 k_{2}\right)\right)\right. \\
& +p_{t}\left(u_{1}-2 k_{1}, 2-\left(1+u_{2}\right)-2 k_{2}\right) \\
& \left.+p_{t}\left(2-\left(1+u_{1}\right)-2 k_{1}, 2-\left(1+u_{2}\right)-2 k_{2}\right)\right] \\
= & f_{t}\left(u_{1}\right) f_{t}\left(u_{2}\right) .
\end{aligned}
$$

Let $s=\left(s_{1}, s_{2}\right)$ is a random vector, uniformly distributed on the set of numbers $\mathcal{I}=\left\{\left(p_{1}, p_{2} / n\right), p_{1}, p_{2}=0, \cdots, n-1\right\}$, and independent random vector $s$ and a random process $\vec{y}(t)$. We introduce the function $F_{t}(\vec{u})=f_{t}(\vec{u}-\vec{s})$, $\vec{u} \in[0,1]^{2}$, then

$$
\begin{aligned}
F_{t}(\vec{u}) & =\frac{1}{n^{2}} \sum_{i \in \mathcal{I}} f_{t}(\vec{u}-\vec{s})=\frac{1}{n^{2}} \sum_{i \in \mathcal{I}} \sum_{k_{1}=-\infty}^{\infty} \sum_{k_{2}=-\infty}^{\infty} p_{t}(\vec{u}-\vec{k}-\vec{i}) \\
& =\frac{1}{n^{2}} \sum_{k_{1}, k_{2}=-\infty}^{\infty} p_{t}(\vec{u}-\vec{k} / n) \\
& =\frac{1}{n} \sum_{k_{1}=-\infty}^{\infty} p_{t}\left(u_{1}-k_{1} / n\right) \frac{1}{n} \sum_{k_{2}=-\infty}^{\infty} p_{t}\left(u_{2}-k_{2} / n\right)=F_{t}\left(u_{1}\right) F_{t}\left(u_{2}\right) .
\end{aligned}
$$

Because of the equality (7) the function $F_{t}(\vec{u})$ has the following properties:

$$
\begin{gathered}
F_{t}(\vec{u})=F_{t}(\vec{u}+\vec{i}), \vec{u} \in[0,1 / n]^{2}, \vec{i} \in \mathcal{I}, \\
F_{t}(\vec{u} / n)=\frac{1}{n^{2}} \sum_{k_{1}, k_{2}=-\infty}^{\infty} p_{t}((\vec{u}-\vec{k}) / n), \vec{u} \in[0,1]^{2} .
\end{gathered}
$$

Let $f(\vec{u})=\left(f\left(u_{1}\right), f\left(u_{2}\right)\right)$, then from Formula (7) it is easy to obtain the equality

$$
\begin{aligned}
F_{t}(\vec{u})-f(\vec{u})= & \left(F_{t}\left(u_{1}\right)-f\left(u_{1}\right)\right)\left(F_{t}\left(u_{2}\right)-f\left(u_{2}\right)\right)+f\left(u_{1}\right)\left(F_{t}\left(u_{2}\right)-f\left(u_{2}\right)\right) \\
& +f\left(u_{2}\right)\left(F_{t}\left(u_{1}\right)-f\left(u_{1}\right)\right) .
\end{aligned}
$$

For a function $\varphi(u)$ defined on the interval $[0,1]$, we introduce the norm $\|\varphi\|=\sup \{|\varphi(u)|, u \in[0,1]\}$. A similar norm is introduced for a function $\varphi(\vec{u})$, defined on the square $[0,1]^{2}$. Let $\Delta_{n}(t)=\left\|F_{t}(u)-f(u)\right\|$, then the following inequality holds

$$
\begin{aligned}
\left\|F_{t}(\vec{u})-f(\vec{u})\right\| \leq & \left\|F_{t}\left(u_{1}\right)-f\left(u_{1}\right)\right\|\left\|F_{t}\left(u_{2}\right)-f\left(u_{2}\right)\right\| \\
& +f\left(u_{2}\right)\left\|F_{t}\left(u_{1}\right)-f\left(u_{1}\right)\right\|+f\left(u_{1}\right)\left\|F_{t}\left(u_{2}\right)-f\left(u_{2}\right)\right\| \\
\leq & \Delta_{n}^{2}(t)+\left(f\left(u_{1}\right)+f\left(u_{2}\right)\right) \Delta_{n}(t) \\
= & \Delta_{n}^{2}(t)+2 \Delta_{n}(t) \rightarrow 0, t \rightarrow \infty .
\end{aligned}
$$

\section{Examples of Random Processes Anomalous Diffusion}

\section{Anomalous diffusion}

Let $y(t), t \geq 0$-homogeneous random process with independent increments, $y(0)=0$. The difference $y(t)-y(\tau), t>\tau \geq 0$ is symmetric on $(-\infty, \infty)$ stable distribution with parameter $a, 1 / 2<a$ and the characteristic function $M \exp (i u[y(t)-y(\tau)])=\exp \left(-(t-\tau)|u|^{1 / a}\right)$.

Process $y(t), t \geq 0$ is describing in [10] anomalous diffusion on an infinite 
straight line. From this definition it follows that this process satisfing equality (4) is self-similar and therefore satisfies the equality (6).

Introduce on $[0,2)$ the binary operation “ $\oplus$ ”, the unary operation of taking inverse element " $\ominus$ ": $u \oplus v=s(u+v), \ominus u=s(-u), u, v \in[0,2)$. It is easy to check that so defined on $[0,2)$ operations generate commutative group $C$ in addition $\oplus$ with the identity element 0 and the inverse $u$ element of $\Theta u$. It is obvious that the mapping $s: E^{1} \rightarrow C$ is a homomorphism of the additive group of real numbers $E^{1}$ on the group $C$, i.e. the fair equalities $s(u+v)=$ $s(u) \oplus s(v), \quad s(-u)=\ominus s(u)$.

Therefore, when $0<t<t+\tau$ we have $s(y(t+\tau))=s(y(t)) \oplus[s(y(t+\tau)-y(t))]$. Consequently, the random process $s(y(t)), t \geq 0$ with independent and homogeneous increments and the distribution density $P_{t}(u)$ is a homogeneous Markov with values in the group $C$, and with the density of the conditional distribution

$$
q_{\tau}(u, v)=p(s(y(t+\tau)))=v / s(y(t)=u)=p(s(y(t+\tau)-y(t))=v-u),
$$

$0 \leq u, v<2$, hence the equality

$$
q_{\tau}(u, v)=q_{\tau}(v, u), 0<\tau, 0 \leq u, v<2
$$

is true. Denote $Q_{\tau}=\inf \left\{q_{\tau}(u, v), 0 \leq u, v<2\right\}$, then

$$
1=\int_{0}^{2} q_{\tau}(u, v) \mathrm{d} v=\int_{0}^{2} q_{\tau}(0, v-u) \mathrm{d} v=\int_{0}^{2} q_{\tau}(0, v) \mathrm{d} v \geq 2 Q_{\tau}>0
$$

and so

$$
0<Q_{\tau} \leq 12, \tau>0 \text {. }
$$

From equality (9), it follows that

$$
\int_{0}^{2} \frac{1}{2} q_{\tau}(u, v) \mathrm{d} v=\frac{1}{2}=\int_{0}^{2} \frac{1}{2} q_{\tau}(v, u) \mathrm{d} v .
$$

Let $P(u), f(u)$ densities of uniform distributions on the intervals $[0,2],[0,1]$, respectively.

Lemma 1. For an arbitrary $t \geq 1$

$$
\left\|f_{t}(u)-f(u)\right\| \leq 2\left(1-2 Q_{1}\right)^{k-1}\left\|P_{1}(u)-P(u)\right\|, k=[t / h] .
$$

Proof. Assuming $\Delta q_{\tau}(u, v)=q_{\tau}(u, v)-Q_{\tau} \geq 0$ and using Formula (10) we obtain for $0 \leq u<2$ :

$$
\begin{aligned}
& \left\|P_{t+\tau}(u)-P(u)\right\| \\
& =\sup \left(\left|\int_{0}^{2} P_{t}(v) q_{\tau}(v, u) \mathrm{d} v-\int_{0}^{2} P(u) q_{\tau}(v, u) \mathrm{d} v\right|, 0 \leq u<2\right) \\
& =\sup \left(\left|\int_{0}^{2} P_{t}(v) \Delta q_{\tau}(v, u) \mathrm{d} v-\int_{0}^{2} P(v) \Delta q_{\tau}(v, u) \mathrm{d} v\right|, 0 \leq u<2\right),
\end{aligned}
$$

that is for $t, \tau \geq 0$

$$
\begin{aligned}
\left\|P_{t+\tau}(u)-P(u)\right\| & \leq\left\|P_{t}(u)-P(u)\right\| \sup \left(\int_{0}^{2} \Delta q_{\tau}(v, u) \mathrm{d} v, 0 \leq u<2\right) \\
& =\left\|P_{\tau}(u)-P(u)\right\|\left(1-2 Q_{\tau}\right) .
\end{aligned}
$$


With the help of Formulas (10), (12) it is easy to obtain that

$$
\left\|P_{t}(u)-P(u)\right\| \leq\left(1-2 Q_{1}\right)^{k-1}\left\|P_{1}(u)-P(u)\right\|, k=[t] .
$$

From Formulas (1), (13) follows the inequality (11). Lemma 2 is proved.

So we obtain geometric by $t$ convergence rate of the density $f_{t}(u)$ to the density of the uniform distribution $f(u)$ with $t \rightarrow \infty$. Moreover, due to (6) with $k=\left[t n^{1 / a}\right], t \rightarrow \infty$

$$
\left\|F_{t}(u)-f(u)\right\| \leq 2\left(1-2 Q_{1}\right)^{\left[t^{\left[n^{1 / q}\right.}\right]-1}\left\|P_{1}(u)-P(u)\right\|=\Delta_{n}(t) \rightarrow 0 .
$$

Hence when we have $n$-periodic initial conditions, the characteristic time mixing with anomalous diffusion is reduced to $n^{1 / a}$ times.

\section{Fractional Brownian motion}

Let $y(t), t \geq 0$ is a fractional Brownian motion [11]. Fractional Brownian motion with Hurst parameter $a$ is Gaussian process with zero mean and covariance function

$$
B_{a}(t, s)=\operatorname{My}(t) y(s)=\frac{\sigma^{2}}{2}\left[|t|^{2 a}+|s|^{2 a}-|t-s|^{2 a} \mid\right], t, s \geq 0, y(0)=0 .
$$

The process of fractional Brownian motion $y(t), t \geq 0$ satisfies the condition of self-similarity (4) and has a symmetric density distributions of $p_{t}(u)$. Using the process $y(t)$ we define reflected from the cut ends [0,1], the process $Y(t), t \geq 0$ by the equation $Y(t)=g(s(y(t)))$.

Lemma 2. When $h=1 / \sigma t^{a}<1, C=18 / \sqrt{2 \pi}$ the following relation is valid:

$$
\sup _{0 \leq u \leq 1}\left|\frac{\partial f_{t}(u)}{\partial u}\right| \leq C h^{3}=\varepsilon \rightarrow 0, t \rightarrow \infty \text {. }
$$

Proof. Fix $u \in[0,1]$ and denote

$$
v_{k}=\frac{k-u}{\sigma t^{a}}, h=v_{k+1}-v_{k}=\frac{1}{\sigma t^{a}}, \psi(v)=v \exp \left(-v^{2} / 2\right) / \sqrt{2 \pi},
$$

remark that

$$
\int_{-\infty}^{\infty} \psi(v) \mathrm{d} v=0
$$

Using Formula (1) and the theorem on the differentiability of a series of the functions compute the derivative

$$
\frac{\partial f_{t}(u)}{\partial u}=\sum_{k=-\infty}^{\infty} \frac{\partial p_{t}(u-k)}{\partial u}=\frac{I}{\sigma^{2} t^{2 a}}, I=\sum_{k=-\infty}^{\infty} \psi\left(v_{k}\right) .
$$

Differentiability of a series of functions standing in the right part of the equality follows from the absolute convergence of the series $I$.

Put $\Delta_{k}(v)=\psi\left(v_{k}\right)-\psi(v), B_{k}=\int_{v_{k}}^{v_{k+1}} \Delta_{k}(v) \mathrm{d} v$ in virtue of Formula (16) the number $I$ can be represented in the form $I=\frac{1}{h} \sum_{k=-\infty}^{\infty} B_{k}$.

Compute the derivative of $\psi(v)$ : 


$$
\begin{aligned}
& \psi^{\prime}(v)=\left(1-v^{2}\right) \exp \left(-v^{2} / 2\right) / \sqrt{2 \pi}, \\
& \psi^{\prime \prime}(v)=\psi(v)\left(v^{2}-3\right), \sup _{-\infty<v<\infty}\left|\psi^{\prime}(v)\right|=1 / \sqrt{2 \pi}
\end{aligned}
$$

Highlight on the real axis $(-\infty, \infty)$ the following segments:

$$
\begin{aligned}
& \mathcal{A}_{1}=(-\infty,-\sqrt{3}], \mathcal{A}_{2}=[-\sqrt{3},-1], \mathcal{A}_{3}=[-1,0], \\
& \mathcal{A}_{4}=[0,1], \mathcal{A}_{5}=[1, \sqrt{3}], \mathcal{A}_{6}=[\sqrt{3}, \infty) .
\end{aligned}
$$

In virtue of (18) run inequalities:

$$
\begin{aligned}
& \psi^{\prime}(v) \leq 0, \psi^{\prime \prime}(v) \leq 0, v \in \mathcal{A}_{1} ; \psi^{\prime}(v) \leq 0, \psi^{\prime \prime}(v) \geq 0, v \in \mathcal{A}_{2}, \\
& \psi^{\prime}(v) \geq 0, \psi^{\prime \prime}(v) \geq 0, v \in \mathcal{A}_{3} ; \psi^{\prime}(v) \geq 0, \psi^{\prime \prime}(v) \leq 0, v \in \mathcal{A}_{4} ; \\
& \psi^{\prime}(v) \leq 0, \psi^{\prime \prime}(v) \leq 0, v \in \mathcal{A}_{5} ; \psi^{\prime}(v) \leq 0, \psi^{\prime \prime}(v) \geq 0, v \in \mathcal{A}_{6} .
\end{aligned}
$$

In accordance with the issued for the segment of $\mathcal{A}_{k}, k=1, \cdots, 6$ inequalities we get:

$$
\begin{aligned}
& -\frac{h}{2} \sum_{6} \psi^{\prime}\left(v_{k+1}\right) \leq S_{6} \leq-\frac{h}{2} \sum_{6} \psi^{\prime}\left(v_{k}\right),-\frac{h}{2} \sum_{5} \psi^{\prime}\left(v_{k}\right) \leq S_{5} \leq-\frac{h}{2} \sum_{5} \psi^{\prime}\left(v_{k+1}\right), \\
& -\frac{h}{2} \sum_{4} \psi^{\prime}\left(v_{k}\right) \leq S_{4} \leq-\frac{h}{2} \sum_{4} \psi^{\prime}\left(v_{k+1}\right),-\frac{h}{2} \sum_{3} \psi^{\prime}\left(v_{k+1}\right) \leq S_{3} \leq-\frac{h}{2} \sum_{3} \psi^{\prime}\left(v_{k}\right), \\
& -\frac{h}{2} \sum_{2} \psi^{\prime}\left(v_{k+1}\right) \leq S_{2} \leq-\frac{h}{2} \sum_{2} \psi^{\prime}\left(v_{k}\right),-\frac{h}{2} \sum_{1} \psi^{\prime}\left(v_{k}\right) \leq S_{1} \leq-\frac{h}{2} \sum_{1} \psi^{\prime}\left(v_{k+1}\right) .
\end{aligned}
$$

Designate $C_{k}=\int_{v_{k+1}}^{v_{k+2}} \psi^{\prime}(v) \mathrm{d} v, D_{k}=\int_{v_{k-1}}^{v_{k}} \psi^{\prime}(v) \mathrm{d} v$ and we deduce from the last inequalities following relationships:

$$
\begin{aligned}
& -\frac{1}{2} \sum_{6} C_{k} \leq S_{6} \leq-\frac{1}{2} \sum_{6} D_{k},-\frac{1}{2} \sum_{5} D_{k} \leq S_{5} \leq-\frac{1}{2} \sum_{5} C_{k}, \\
& -\frac{1}{2} \sum_{4} D_{k} \leq S_{4} \leq-\frac{1}{2} \sum_{4} C_{k},-\frac{1}{2} \sum_{3} C_{k} \leq S_{3} \leq-\frac{1}{2} \sum_{3} D_{k}, \\
& -\frac{1}{2} \sum_{2} C_{k} \leq S_{2} \leq-\frac{1}{2} \sum_{2} D_{k},-\frac{1}{2} \sum_{1} D_{k} \leq S_{1} \leq-\frac{1}{2} \sum_{1} C_{k} .
\end{aligned}
$$

From them it is easy to obtain:

$$
\begin{gathered}
-\frac{1}{2} \int_{\sqrt{3}+3 h}^{\infty} \psi^{\prime}(v) \mathrm{d} v \leq S_{6} \leq-\frac{1}{2} \int_{\sqrt{3}}^{\infty} \psi^{\prime}(v) \mathrm{d} v, \\
-\frac{1}{2} \int_{1+h}^{\sqrt{3}-3 h} \psi^{\prime}(v) \mathrm{d} v \leq S_{5} \leq-\frac{1}{2} \int_{1+2 h}^{\sqrt{3}} \psi^{\prime}(v) \mathrm{d} v, \\
-\frac{1}{2} \int_{0}^{1-2 h} \psi^{\prime}(v) \mathrm{d} v \leq S_{4} \leq-\frac{1}{2} \int_{3 h}^{1-h} \psi^{\prime}(v) \mathrm{d} v, \\
-\frac{1}{2} \int_{-1}^{0} \psi^{\prime}(v) \mathrm{d} v \leq S_{3} \leq-\frac{1}{2} \int_{-1+h}^{-2 h} \psi^{\prime}(v) \mathrm{d} v, \\
-\frac{1}{2} \int_{-\sqrt{3}+3 h}^{-1-h} \psi^{\prime}(v) \mathrm{d} v \leq S_{2} \leq-\frac{1}{2} \int_{-\sqrt{3}}^{-1-2 h} \psi^{\prime}(v) \mathrm{d} v, \\
-\frac{1}{2} \int_{-\infty}^{-\sqrt{3}-3 h} \psi^{\prime}(v) \mathrm{d} v \leq S_{1} \leq-\frac{1}{2} \int_{-\infty}^{-\sqrt{3}} \psi^{\prime}(v) \mathrm{d} v .
\end{gathered}
$$


Therefore, we have:

$$
\begin{gathered}
\frac{\psi(\sqrt{3}+3 h)}{2} \leq S_{6} \leq \frac{\psi(\sqrt{3})}{2}, \\
\frac{\psi(1+h)-\psi(\sqrt{3}-3 h)}{2} \leq S_{5} \leq \frac{\psi(1+2 h)-\psi(\sqrt{3})}{2}, \\
\frac{-\psi(1-2 h)}{2} \leq S_{4} \leq \frac{\psi(3 h)-\psi(1-h)}{2}, \\
\frac{\psi(-1)}{2} \leq S_{3} \leq \frac{\psi(-1+h)-\psi(-2 h)}{2}, \\
\frac{-\psi(-1-h)+\psi(-\sqrt{3}+3 h)}{2} \leq S_{2} \leq \frac{-\psi(-1-2 h)+\psi(-\sqrt{3})}{2}, \\
\frac{-\psi(-\sqrt{3}-3 h)}{2} \leq S_{1} \leq \frac{-\psi(-\sqrt{3})}{2}
\end{gathered}
$$

and so the sum $S=\sum_{k=1}^{6} S_{k}$ satisfies the inequalities:

$$
\begin{aligned}
& \frac{\psi(\sqrt{3}+3 h)-\psi(\sqrt{3}-3 h)+\psi(1+h)-\psi(1-2 h)}{2} \\
& +\frac{\psi(-1)-\psi(-1-h)+\psi(-\sqrt{3}+3 h)-\psi(-\sqrt{3}-3 h)}{2} \\
& \leq S \leq \frac{\psi(1+2 h)-\psi(1-h)+\psi(3 h)-\psi(-2 h)+\psi(-1+h)-\psi(-1-2 h)}{2}
\end{aligned}
$$

how do we get that

$$
|S| \leq \frac{\max (3 h+5 h+3 h, 6 h+3 h+h+6 h)}{2 \sqrt{2 \pi}}=8 h / \sqrt{2 \pi} .
$$

Because of Formula (18), the resultant inequality $\left|B_{k}\right| \leq h^{2} / 2 \sqrt{2 \pi}$ and the definition of the sums $\sum_{k}, k=1, \cdots, 6$, we find:

$$
\begin{aligned}
|I-S| & \leq \frac{1}{h}\left(\sum_{\sqrt{3}-2 h \leq v_{k} \leq \sqrt{3}+h}+\sum_{1-2 h \leq v_{k} \leq 1+h}+\sum_{-2 h \leq v_{k} \leq h}+\sum_{-1-2 h \leq v_{k} \leq-1+h}+\sum_{-\sqrt{3}-2 h \leq v_{k} \leq-\sqrt{3}+h}\right) B_{k} \\
& \leq 10 h / \sqrt{2 \pi} .
\end{aligned}
$$

Therefore, the following inequality holds $|I| \leq 18 h / \sqrt{2 \pi}$, which leads to the relation (11). Lemma 2 is proved.

Theorem 3. If $h<1$ the following inequality holds

$$
\left\|f(u)-f_{t}(u)\right\| \leq 3 \varepsilon \rightarrow 0, t \rightarrow \infty .
$$

Proof. Because of Formula (1) equalities $f_{t}(u)=f(u)=0, u \notin[0,1]$ take place. Let $\delta_{t}(u)=f_{t}(u)-f_{1}(0), u \in[0,1]$. In Lemma 2 the following inequality holds $\left|\delta_{t}(u)\right| \leq(u+1) \varepsilon, u \in[0,1]$, therefore

$$
1=\int_{0}^{1} f_{t}(u) \mathrm{d} u=f_{t}(0)+\int_{0}^{1} \delta_{t}(u) \mathrm{d} u
$$

and hence $\left|f_{t}(0)-f(u)\right| \leq \varepsilon$. Here we come to the statement of Theorem 3 . 
Because of Formula (6) from inequality (19) we have

$$
\begin{aligned}
\left\|F_{t}(u)-f(u)\right\| & =\left\|F_{t}(u-1 / 2 n)-f(u)\right\|=\left\|f_{t n^{1 / a}}-f(u)\right\| \\
& \leq 3 C \sigma^{3} t^{3 a} n^{3}=\Delta_{n}(t) \rightarrow 0, t \rightarrow \infty .
\end{aligned}
$$

Hence for $n$-periodic initial conditions the characteristic time mixing under fractional Brownian motion is reduced to $n^{a}$ times.

\section{Conclusions}

Obtained in the present work, an upper estimate of the convergence rate of the density function of a multidimensional fractional Brownian motion with reflection at the boundaries of a square is not exponential as in the usual Brownian motion or a stable process with independent increments. Apparently this is due to the fact that the multidimensional fractional Brownian motion models the processes with chaotic behavior [1].

In the work [12], it is considered a model of fractional Brownian motion with dependent components. However, this model fails to obtain the corresponding results in the case of periodic initial conditions. But in the case of independent components multidimensional Brownian motion satisfies the statement of Theorem 3.

\section{Acknowledgements}

The paper is supported by RFBR, project 17-07-00177.

\section{References}

[1] Chen, S., Yu, L.P., Ren, J.L., Xie, X., Li, X.P., Xu, Y., Zhao, G.F., Li, P.Z., Yang, F.Q., Ren, Y. and Liaw, P.K. (2016) Self-Similar Random Process and Chaotic Behavior. In Serrated Flow of High Entropy Alloys. Scientific Reports, 6, Article Number: 29798. https://doi.org/10.1038/srep29798

[2] Caroll, R., Lee, C., Tsai, C.-W., Yeh, J.-W., Antonaglia, J., Brinkman, B.A.W., et al. (2015) Experiments and Model for Serration Statistics in Low-Entropy, MediumEntropy, and High-Entropy Alloys. Scientific Reports, 5, Article Number: 16997. https://doi.org/10.1038/srep16997

[3] Zhang, Z.J., et al. (2015) Experiments and Model for Serration Statistics in Low-Entropy, Medium Entropy Alloys. Nat Commun, 6. PMC free article.

[4] Youssef, K.M., Zaddach, A.J., Niu, C.N., Irving, D.L. and Koch, C.C. (2015) A Novel Low Density, High-Hardness, High Entropy Alloy with Close Packed Single-Phase Nanocrystalline Structures. Materials Research Letters, 3, No. 2.

[5] Tsitsiashvili, G.Sh. (2015) Characteristic Time of Diffusive Mixing in Cube with Reflecting Edges. American Journal of Modern Physics, 6, 81-87.

[6] Tsitsiashvili, G.Sh. (2013) Anomalous Diffusion on Finite Interval. Journal of Mathematical Sciences, 191, 582-587. https://doi.org/10.1007/s10958-013-1342-5

[7] Tsitsiashvili, G.Sh. (2017) Convergence of Fractional Brownian Motion with Reflections to Uniform Distribution. Applied Mathematical Sciences, 11, 2103-2112.

[8] Vladimirov, V.S. (1967) Equations of Mathematical Physics. Nauka, Moscow. (In Russian) 
[9] Embrechts, P. and Maejima, M. (2000) An Introduction to the Theory of Self-Similar Stochastic Processes. International Journal of Modern Physics B, 14, 1399. https://doi.org/10.1142/S0217979200001047

[10] Uchaikin, V.V. (2002) Multidimensional Symmetric Anomalous Diffusion. Chemical Physics, 284, 507-520. https://doi.org/10.1016/S0301-0104(02)00676-6

[11] Mikosch, T., et al. (2002) Is Network Traffic Approximated by Stable Levy Motion or Fractional Brownian Motion? Annals of Applied Probability, 12, 23-68.

[12] Tsitsiashvili, G.Sh. (2017) Multivariate Fractional Brownian Motion in Cube with Reflecting Edges. Applied Mathematical Sciences, 11, 2315-2324. 